\title{
Hipertensão no grande idoso: tratar ou não tratar?
}

Teresa Moura Bastos, ${ }^{*}$ Sofia Azenha, ${ }^{* *}$ Tânia Dias***

\section{RESUMO}

Introdução: A hipertensão arterial é o fator de risco cardiovascular mais prevalente. Sabe-se que a diminuição da pressão arterial (PA) em hipertensos com $\leq 65$ anos reduz a ocorrência de eventos cardiovasculares e morte. Relativamente ao grande idoso ( $\geq 80$ anos), ainda há poucos estudos que possam assegurar o mesmo consenso.

Objetivos: Rever se existe evidência sobre se o tratamento anti-hipertensor conduz a diminuição de eventos cardiovasculares major, bem como a redução da mortalidade no grande idoso.

Fontes de dados: National Guideline Clearinghouse, Guideline Finder, Canadian Medical Association, The Cochrane Database, DARE, Bandolier, MEDLINE/PubMed e Índex de Revistas Médicas Portuguesas.

Métodos de revisão: Pesquisa em sites de medicina baseada na evidência; entre janeiro de 2004 e agosto de 2014 ; línguas inglesa, espanhola ou portuguesa; palavras-chave: Hypertension/therapy AND Aged, 80 and over.

Resultados: Oito artigos cumpriam os critérios de inclusão. Segundo as normas, o tratamento anti-hipertensor reduz a incidência de acidente vascular cerebral (AVC) e morbilidade cardiovascular e aumenta a mortalidade por outras causas (FR C). Se há boa condição física e psicológica, os valores de PA devem rondar os 140-150mmHg (FR B); caso contrário, o tratamento deve ser individualizado (FR C). As meta-análises corroboram a redução do risco de AVC, eventos cardiovasculares e insuficiência cardíaca (IC) (NE 2). Os ensaios clínicos revelam benefício na redução da PA até 150/80mmHg (NE 1); valores inferiores a este associam-se a maior risco de morte cerebrovascular e IC (NE 2).

Conclusão: O benefício global do tratamento no grande idoso é questionável, pois embora esteja associado a uma redução dos eventos cardiovasculares quanto à mortalidade total, bem como à mortalidade específica por causa, os resultados são heterogéneos (FR B). Salienta-se, contudo, o facto de a redução da mortalidade total ser conseguida nos ensaios com menores reduções da PA e terapêuticas menos intensivas (FR B).

Palavras-chave: Hipertensão; Tratamento; Grande Idoso.

\section{INTRODUÇÃO}

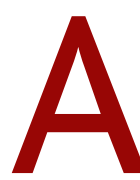
hipertensão arterial (HTA) é o fator de risco cardiovascular mais prevalente e afeta até $60 \%$ dos utentes com idade $\geq 60$ anos e $75 \%$ daqueles com idade $\geq 85$ anos. $^{1}$

Existe evidência científica de que a diminuição da pressão arterial (PA) em utentes hipertensos com idade $\leq 65$ anos reduz significativamente a ocorrência de eventos cardiovasculares e morte. No que diz respeito ao

* Médica interna de Medicina Geral e Familiar.

Unidade de Cuidados de Saúde Primários Sul, Centro de Saúde de Oliveira de Azeméis, Agrupamento de Centros de Saúde Entre Douro e Vouga II, Administração Regional do Norte.

**Médica especialista de Medicina Geral e Familiar. Unidade de Saúde Familiar Famílias, Centro de Saúde de Santa Maria da Feira, Agrupamento de Centros de Saúde Entre Douro e Vouga I, Administração Regional do Norte.

***Médica interna de Medicina Geral e Familiar. Unidade de Saúde Familiar Famílias, Centro de Saúde de Santa Maria da Feira, Agrupamento de Centros de Saúde Entre Douro e Vouga I, Administração Regional do Norte. grande idoso, esta temática ainda não é consensual. ${ }^{2-3}$ Sabe-se que o risco de acidente vascular cerebral (AVC) aumenta para valores de $\mathrm{PA} \geq 115 / 75 \mathrm{mmHg}$; contudo, esta associação diminui com o aumento da idade. ${ }^{4}$

De facto, os hipertensos com idade $\geq 80$ anos (grande idoso) têm vindo a ser pouco considerados em ensaios clínicos e, desta forma, a base científica utilizada para o seu tratamento é extrapolada de utentes muito mais novos. ${ }^{1}$ Permanece, assim, a dúvida relativamente aos valores alvo de PA a atingir nesta faixa etária, bem como a incerteza de como uma redução excessiva da PA possa conduzir a um aumento de eventos cardiovasculares, como AVC, quedas, problemas cognitivos e sintomas depressivos, levando consequentemente ao aumento da morbilidade e mortalidade nesta faixa etária. ${ }^{1}$ Diversos estudos referem que há mesmo uma relação inversa entre o valor da PA e a 
mortalidade para utentes $\geq 80$ anos, correlacionando-se com o aumento das complicações associadas a esta redução. ${ }^{4}$

Grande parte dos grandes idosos hipertensos encontram-se sem tratamento, com controlo inadequado da PA ou sob tratamento com fármacos menos adequados..$^{3-4} \mathrm{~A}$ agravar ainda mais esta problemática surge o facto de a HTA ser um diagnóstico frequente no grande idoso, faixa etária que está progressivamente a aumentar nos países desenvolvidos. ${ }^{3-4}$ Estima-se que, em 2030, cerca de $20 \%$ da população mundial terá idade igual ou superior a 65 anos. ${ }^{1}$

O objetivo do presente estudo é rever se, na faixa etária do grande idoso, existe evidência de que o tratamento anti-hipertensor conduz à diminuição de eventos cardiovasculares major, bem como à redução da mortalidade.

\section{MÉTODOS}

Foi realizada uma pesquisa bibliográfica a 4 de agosto de 2014 com os termos MeSH e operador booleano: Hypertension/therapy AND Aged, 80 and over.

As bases de dados pesquisadas incluem: National Guideline Clearinghouse, Guideline Finder, Canadian Medical Association, The Cochrane Database, DARE, Bandolier, MEDLINE/PubMed e Índex de Revistas Médicas Portuguesas. Pesquisaram-se ainda as referências bibliográficas dos artigos originais e Normas de Orientação Clínica selecionados. Incluíram-se os artigos publicados nos últimos dez anos (janeiro de 2004 a agosto de 2014) em língua portuguesa, inglesa ou espanhola que respeitassem os critérios de elegibilidade a seguir especificados:

- População: hipertensos com idade igual ou superior a 80 anos;

- Intervenção: tratamento anti-hipertensor;

- Comparação: sem tratamento ou placebo;

- Outcome (orientado para o utente): diminuição do número de eventos cardiovasculares major e da mortalidade.

Considerou-se como grande idoso o utente com idade $\geq 80$ anos por ser essa a definição da Organização Mundial da Saúde (OMS) para a maioria dos países desenvolvidos e por ser a considerada nos termos $\mathrm{MeSH}$ dos motores de busca utilizados.

Definiram-se como critérios de exclusão:
- Presença de hipertensos noutras faixas etárias para além da definida nos critérios de elegibilidade;

- Não utilização de tratamento farmacológico na redução de PA;

- Comparação entre diversos tipos de tratamentos farmacológicos para a redução de PA;

- O artigo não corresponder a nenhum dos seguintes tipos: Norma de Orientação Clínica (NOC), meta-análise (MA), artigos de revisão (AR) orientados para o utente, ensaio clínico randomizado e controlado (ECR), estudo de coorte e estudo de caso-controlo. Para avaliação dos níveis de evidência (NE) e atribuição de forças de recomendação (FR) foi usada a escala Strenght of Recommendation Taxonomy (SORT), da American Family Physician.

\section{RESULTADOS}

Na pesquisa bibliográfica inicial obtiveram-se 225 artigos, sendo que se reviram oito artigos completos (duas MA, duas NOC, dois ECR e dois estudos de coorte). A seleção dos mesmos encontra-se descrita no fluxograma I e os seus resultados estão sumariados no quadro I, II, III e IV.

Numa das meta-análise incluídas, Bejan-Angoulvant, et al, ${ }^{2}$ foram analisados oito ensaios clínicos randomizados que comparavam o tratamento anti-hipertensor com o placebo no grande idoso e tinham como outcome principal a mortalidade total e, como outcomes secundários, o AVC, os eventos cardiovasculares, a insuficiência cardíaca (IC) e a mortalidade específica para a causa. Segundo esta MA, o tratamento anti-hipertensor reduziu significativamente o risco de AVC, eventos cardiovasculares e IC (NE 2). Relativamente à mortalidade total, os resultados dos ensaios clínicos incluídos foram heterogéneos, pelo que não foi possível tirar conclusões (NE 2). Contudo, a redução da mortalidade total foi conseguida nos ensaios com menor redução da PA e terapêutica farmacológica menos intensiva (NE 2). No que diz respeito à mortalidade específica por causa, não houve diferenças estatisticamente significativas entre tratados e não tratados (NE 2).

Na outra meta-análise incluída, Law, et al, ${ }^{5}$ foram analisados 108 ensaios clínicos randomizados que comparavam o tratamento anti-hipertensor com o placebo. Esta MA tinha como outcome a doença coronária 


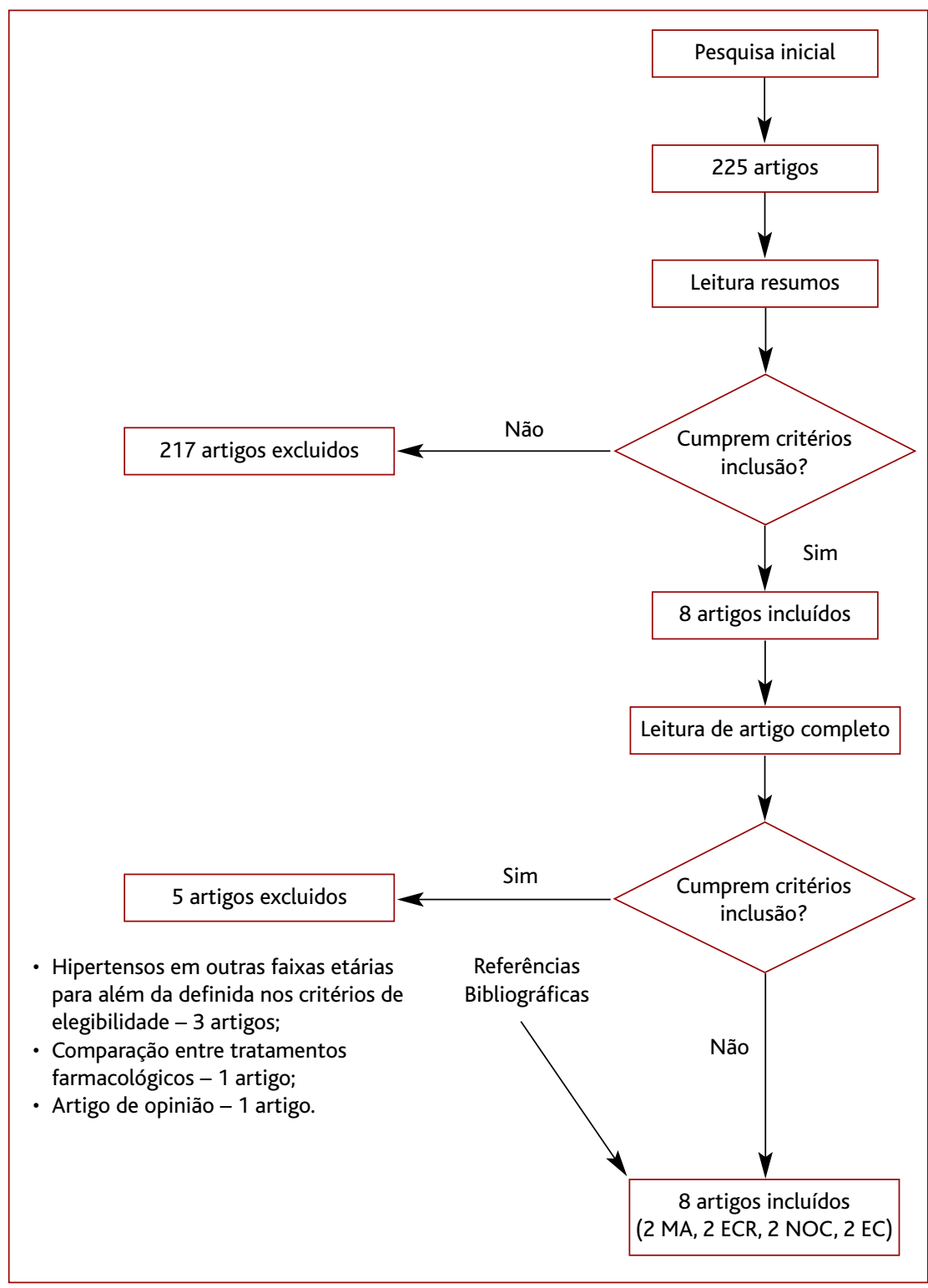

Fluxograma I. Processo de seleção dos artigos.

Legenda: MA - meta-análise; ECR - ensaio clínico controlado e randomizado; NOC - norma de orientação clínica; EC - estudo de coorte.

e o enfarte agudo do miocárdio. Verificou-se que o tratamento anti-hipertensor reduziu significativamente o risco de doença coronária e enfarte agudo do miocárdio no grande idoso e que esse benefício foi tanto maior quanto maior o valor de PAs e PAd inicial (NE 1).

O estudo HYVET, ${ }^{4}$ um ensaio clínico aleatorizado, controlado e duplamente cego com 3.845 hipertensos de $\geq 80$ anos, desenvolvido na Europa, China, Australá- sia e Tunísia, comparou o tratamento com indapamida de libertação prolongada isolada (ou associação indapamida + perindopril) com o placebo. Este estudo teve como outcome primário o AVC fatal e não fatal e, como outcomes secundários, a morte de causa cerebrovascular, morte de qualquer tipo de causa e a IC. Os resultados obtidos mostram que o tratamento anti-hipertensor do grande idoso (indapamida com ou sem perindopril) até valores alvo de 150/80 mmHg foi benéfico, devido a uma diminuição estatisticamente significativa do risco de AVC fatal e não fatal (30\%), morte de causa cerebrovascular (39\%), morte de qualquer causa (21\%) e risco de IC (23\%) (NE 1).

Outro ensaio clínico desenvolvido em $2011,{ }^{6}$ que constituiu uma extensão do estudo HYVET, teve como objetivo determinar se o grande idoso obtinha benefícios com o tratamento anti-hipertensor precoce. Assim, os participantes sob anti-hipertensor no estudo HYVET continuaram com tratamento farmacológico e os que estavam a fazer placebo iniciaram anti-hipertensor. Os resultados obtidos foram uma menor mortalidade total e eventos cardiovasculares nos participantes sob terapêutica anti-hipertensora precoce, não havendo contudo diferenças na ocorrência de AVC ou eventos cardiovasculares entre os dois grupos (NE 1). Os investigadores concluíram que as diferenças observadas na redução da mortalidade total e cardiovascular suportavam os benefícios e a necessidade de tratamento anti-hipertensor precoce e de longa duração (NE 1).

A guideline de 2011 da American College of Cardiology Foundation/American Heart Association (ACCF/ $\mathrm{AHA})^{7}$ referiu que o tratamento anti-hipertensor reduz 


\section{QUADRO I. Meta-análises}

\begin{tabular}{|c|c|c|c|}
\hline Referência (ano) & Metodologia & Resultados & $\mathrm{NE}$ \\
\hline $\begin{array}{l}\text { Bejan-Angoulvant } \\
\text { (2010) } \\
\text { [1] }\end{array}$ & $\begin{array}{l}\text { Meta-análise de oito ECR ( } \geq 80 \\
\text { anos; anti-hipertensor vs. } \\
\text { placebo) } \\
\text { Outcome principal: mortalidade } \\
\text { total } \\
\text { Outcomes secundários: AVC, } \\
\text { eventos cardiovasculares, IC, } \\
\text { mortalidade específica por } \\
\text { causa }\end{array}$ & $\begin{array}{l}\text { O tratamento anti-hipertensor reduz significativamente o risco } \\
\text { de AVC, eventos cardiovasculares e IC } \\
\text { - Há grande heterogeneidade dos resultados no que concerne à } \\
\text { mortalidade total, pelo que não são possíveis conclusões } \\
\text { - A redução da mortalidade total foi conseguida nos ensaios com } \\
\text { menor redução da pressão arterial e terapêutica farmacológica } \\
\text { menos intensiva } \\
\text { - A mortalidade específica por causa não difere entre tratados e } \\
\text { não tratados }\end{array}$ & 2 \\
\hline $\begin{array}{l}\text { Law } \\
(2009) \\
{[5]}\end{array}$ & $\begin{array}{l}\text { Meta-análise de } 108 \text { ECR } \\
\text { (40-49; 50-59; 60-69; 70-79; } \\
\text { 80-89; anti-hipertensor vs. } \\
\text { placebo) } \\
\text { Outcome: doença coronária e } \\
\text { enfarte agudo do miocárdio }\end{array}$ & $\begin{array}{l}\text { - No grande idoso, o tratamento anti-hipertensor reduz } \\
\text { significativamente o risco de doença coronária e enfarte agudo } \\
\text { do miocárdio, sendo esse benefício tanto maior quanto maior é } \\
\text { o valor de PAs e PAd inicial }\end{array}$ & 1 \\
\hline
\end{tabular}

Legenda: AVC - acidente vascular cerebral; ECR - ensaio clínico controlado e randomizado; IC - insuficiência cardíaca; PAd - pressão arterial diastólica; PAs - pressão arterial sistólica; vs. - versus.

\section{QUADRO II. Ensaios clínicos aleatorizados e controlados}

\begin{tabular}{|c|c|c|c|c|}
\hline $\begin{array}{l}\text { Referência } \\
\text { (ano) }\end{array}$ & $\begin{array}{l}\text { Tipo de estudo } \\
\text { e amostra }\end{array}$ & Intervenção & Resultados & NE \\
\hline $\begin{array}{l}\text { Beckett } \\
(2008) \\
{[4]}\end{array}$ & $\begin{array}{l}\text { Ensaio clínico } \\
\text { aleatorizado, } \\
\text { controlado e } \\
\text { duplamente cego. } \\
n=3845 \text { hipertensos } \\
\text { com } \geq 80 \text { anos e PAs } \\
\geq 160 \text {. } \\
\text { Seguimento médio: } \\
1,8 \text { anos }\end{array}$ & $\begin{array}{l}\text { Grupo Tratamento }(\mathrm{n}=1933) \text { : } \\
\text { Indapamida }(1,5 \mathrm{mg}) \text {; perindopril } \\
(2-4 \mathrm{mg}) \text { adicionado para atingir meta } \\
\text { 150/80mmHg. } \\
\text { Grupo Controlo }(\mathrm{n}=1912) \text { : placebo. } \\
\text { Outcome primário: AVC fatal e não fatal. } \\
\text { Outcomes secundários: morte de causa } \\
\text { cerebrovascular, morte de qualquer } \\
\text { tipo de causa e IC. }\end{array}$ & $\begin{array}{l}\text { Tratamento grande idoso indapamida com } \\
\text { ou sem perindopril, até } 150 / 80 \mathrm{mmHg} \text {, é } \\
\text { benéfico, dado diminuição (estatisticamente } \\
\text { significativa) do risco de AVC fatal e não } \\
\text { fatal }(30 \%) \text {, morte de causa cerebrovascular } \\
\text { (39\%), morte de qualquer causa ( } 21 \% \text { ) e } \\
\text { risco de IC }(23 \%) \text {. }\end{array}$ & 1 \\
\hline $\begin{array}{l}\text { Beckett } \\
(2011) \\
{[6]}\end{array}$ & $\begin{array}{l}\text { Extensão do ECA } \\
\text { duplo-cego HYVET } \\
\text { Período de estudo: } \\
\text { um ano }\end{array}$ & $\begin{array}{l}\text { Participantes sob anti-hipertensor no } \\
\text { HYVET permaneceram a fazer o fármaco. } \\
\text { Participantes sob placebo iniciaram } \\
\text { anti-hipertensor. } \\
\text { Tratamento: Indapamida } 1,5 \mathrm{mg} \\
\text { (perindopril } 2-4 \mathrm{mg} \text {, se necessário). } \\
\text { Alvo terapêutico de } 150 / 80 \mathrm{mmHg} \text {. } \\
\text { Outcome primário: AVC. } \\
\text { Outcome secundário: mortalidade } \\
\text { total e eventos cardiovasculares. }\end{array}$ & $\begin{array}{l}\text { Menor mortalidade total e cardiovascular } \\
\text { nos participantes sob terapêutica desde } \\
\text { HYVET. } \\
\text { Sem diferenças na ocorrência de AVC ou } \\
\text { eventos cardiovasculares entre os dois } \\
\text { grupos. As diferenças observadas na redução } \\
\text { da mortalidade total e cardiovascular } \\
\text { suportam os benefícios e necessidade de } \\
\text { tratamento anti-hipertensor precoce e de } \\
\text { longa duração. }\end{array}$ & 1 \\
\hline
\end{tabular}

Legenda: AVC - acidente vascular cerebral; IC - insuficiência cardíaca; PAs - pressão arterial sistólica. 


\section{QUADRO III. Normas de orientação clínica}

\begin{tabular}{|c|c|c|c|}
\hline Referência & Ano & Recomendações & FR \\
\hline $\begin{array}{l}\text { ACCF/ AHA Consensus on } \\
\text { hypertension in the elderly } \\
\text { (circulation) [7] }\end{array}$ & 2011 & $\begin{array}{l}\text { - Reduz a incidência de acidente vascular cerebral e morbilidade cardiovascular } \\
\text { - Aumenta a mortalidade por outras causas de morte } C\end{array}$ & C \\
\hline $\begin{array}{l}\text { ESH/ESC Guidelines for the } \\
\text { management of arterial } \\
\text { hypertension [8] }\end{array}$ & 2013 & $\begin{array}{l}\text { - } \geq 80 \text { anos e PAs } \geq 160 \mathrm{mmHg} \text { recomenda-se a redução para valores entre } 140 \\
\text { e } 150 \mathrm{mmHg} \text {, caso estes tenham boa condição física e psicológica } \\
\text { - Em grandes idosos fragilizados recomenda-se individualizar o tratamento } \\
\text { anti-hipertensor com base na monitorização das consequências clínicas } \\
\text { - A continuidade do tratamento bem tolerado deve ser considerada quando o } \\
\text { utente se torna octogenário }\end{array}$ & $\begin{array}{l}\text { B } \\
\text { C } \\
\text { C }\end{array}$ \\
\hline
\end{tabular}

Legenda: ACCF - American College of Cardiology Foundation; AHA - American Heart Association; ESC - European Society of Cardiology; ESH - European Society of Hypertension.

\begin{tabular}{|c|c|c|c|c|}
\hline $\begin{array}{l}\text { Referência } \\
\text { (ano) }\end{array}$ & Tipo de estudo e amostra & Intervenção & Resultados & NE \\
\hline $\begin{array}{l}\text { Oates } \\
(2007) \\
{[3]}\end{array}$ & $\begin{array}{l}\text { Estudo de coorte retrospetivo } \\
\text { Coorte: } 471 \text { hipertensos } \geq 80 \text { anos } \\
\text { Período de estudo: } 5 \text { anos }\end{array}$ & $\begin{array}{l}\text { Coorte do estudo Veterans AFFAIRS } \\
\text { Outcome primário: morte durante } \\
\text { período de estudo } \\
\text { Outcome secundário: pressão } \\
\text { arterial base, se faz ou não } \\
\text { medicação, tipo de medicação, } \\
\text { outros diagnósticos }\end{array}$ & $\begin{array}{l}\text { Os utentes com maior pressão } \\
\text { sanguínea (PAs } \geq 139 \mathrm{mmHg} \text { e PAd } \\
\geq 89 \mathrm{mmHg} \text { ) tinham uma taxa de } \\
\text { mortalidade significativamente } \\
\text { inferior aos utentes com pressão } \\
\text { sanguínea abaixo deste valor }\end{array}$ & 2 \\
\hline $\begin{array}{l}\text { Lloyd-Jones } \\
\text { (2005) } \\
{[2]}\end{array}$ & $\begin{array}{l}\text { Estudo de coorte retrospetivo } \\
\text { Coorte: } 5.296 \text { hipertensos }<60 ; \\
{[60 ; 79] ; \geq 80 \text { anos }} \\
\text { Período de estudo: } 6 \text { anos }\end{array}$ & $\begin{array}{l}\text { Coorte do estudo Framingham } \\
\text { Heart } \\
\text { Outcomes primários: prevalência } \\
\text { HTA, do controlo de HTA } \\
\text { (comparados entre faixas etárias) } \\
\text { Outcomes secundários: eventos } \\
\text { cardiovasculares major, IC, doença } \\
\text { coronária }\end{array}$ & $\begin{array}{l}\text { No grupo de utentes } \geq 80 \text { anos o } \\
\text { risco de ocorrer um evento } \\
\text { cardiovascular major, doença } \\
\text { coronária ou insuficiência cardíaca } \\
\text { é inferior quando os valores de PA } \\
\text { se encontram entre PAs } \\
\text { 140-159mmHg e PAd } \\
90-100 \mathrm{mmHg} \text {, comparativamente } \\
\text { a valores inferiores (PAs } \\
<140 \mathrm{mmHg} \text { e PAd <90mmH) e } \\
\text { superiores (PAs >160mmHg e } \\
\text { PAd >100mmH) de PA. }\end{array}$ & 2 \\
\hline
\end{tabular}

Legenda: HTA - hipertensão arterial; PA - pressão arterial; PAd - pressão arterial diastólica; PAs - pressão arterial sistólica.

a incidência de AVC e a morbilidade cardiovascular (FR $\mathrm{C}$ ), apesar de aumentar a mortalidade por outras causas de morte (FR C).

Segundo a guideline da European Society of Hyper-
tension/European Society of Cardiology (ESH/ESC), de $2013,{ }^{8}$ no grande idoso com PAs $\geq 160 \mathrm{mmHg}$ recomenda-se a redução da PA para valores entre 140 e $150 \mathrm{mmHg}$, caso estes disponham de boa condição fí- 
sica e psicológica (FR B). Em grandes idosos fragilizados recomenda-se individualizar o tratamento anti-hipertensor com base na monitorização das consequências clínicas (FR C). Contudo, a continuidade de um tratamento bem tolerado deve ser considerada quando o utente se torna octogenário (FR C).

Um estudo de coorte retrospetivo com 471 hipertensos grandes idosos, ${ }^{1}$ cujo outcome primário foi a morte durante o período de estudo (cinco anos), referiu que os utentes com maior pressão sanguínea (PAs $\geq 139 \mathrm{mmHg}$ e PAd $\geq 89 \mathrm{mmHg}$ ) tinham uma taxa de mortalidade inferior aos utentes com pressão sanguínea abaixo deste valor (NE 2).

Outro estudo de coorte ${ }^{3}$ retrospetivo com 5.296 hipertensos obteve os seguintes resultados: no grupo de utentes $\geq 80$ anos o risco de ocorrer um evento cardiovascular major, doença coronária ou insuficiência cardíaca foi inferior quando os valores de PA se encontravam entre PAs 140-159mmHg e PAd 90-100mmHg, comparativamente a valores inferiores ( $\mathrm{PAs}<140 \mathrm{mmHg}$ e PAd $<90 \mathrm{mmHg}$ ) e superiores (PAs $>160 \mathrm{mmHg}$ e PAd $>100 \mathrm{mmHg}$ ) de PA (NE 2).

\section{CONCLUSÕES}

Com esta revisão podemos concluir que o benefício global do tratamento no grande idoso é questionável, pois embora esteja associado a uma redução dos eventos cardiovasculares, AVC e IC (FR B) quanto à mortalidade total, bem como mortalidade específica por outras causas, os resultados são heterogéneos, pelo que há necessidade de realização de mais estudos para a obtenção de conclusões mais robustas (FR B). Salienta-se, contudo, o facto de a redução da mortalidade total ser conseguida nos ensaios com menores reduções da PA e em que as terapêuticas foram menos intensivas (FR B).

Também podemos concluir que a evidência científica vai no sentido do benefício da redução da PAs para valores entre $140-150 \mathrm{mmHg}$ no grande idoso hipertenso, com PAs inicial $\geq 160 \mathrm{mmHg}$ e com bom estado geral (FR B). No que concerne ao grande idoso fragilizado, a evidência leva-nos a concluir que a terapêutica deve ser individualizada, com base nas comorbilidades e evolução clínica do utente (FR C). Contudo, não há necessidade de interromper um tratamento de sucesso e bem tolerado quando o utente atinge os 80 anos, recomendando-se a individualização do mesmo, de acordo com o utente em causa, suas comorbilidades e fatores de risco cardiovasculares associados (FR C).

Consideram-se, como limitações desta revisão, o reduzido número de estudos nesta faixa etária, os utentes incluídos nos mesmos serem habitualmente saudáveis, o facto de a definição de grande idoso não ser mundialmente consensual e a inclusão nesta revisão de estudos metodologicamente diferentes, nomeadamente quanto ao desenho de estudo, tamanho amostral, outcomes e tipo de fármacos utilizados.

Considerando a fragilidade dos estudos e guidelines disponíveis e o desinvestimento científico em relação ao tratamento do grande idoso hipertenso, torna-se essencial desenvolver estudos (como a presente revisão) que sumariem a evidência existente, de forma a podermos tratar de forma segura os nossos utentes, evitando, assim, as consequências do excesso de intervencionismo médico. Cabe-nos a nós, médicos de família, através da prevenção quaternária, reforçar a análise do processo de decisão em situações de incerteza, utilizando a epidemiologia clínica e a medicina baseada na evidência para analisar os ganhos potenciais de um tratamento face aos riscos acrescidos da sobremedicalização.

\section{AGRADECIMENTOS}

As autoras gostariam de agradecer à $\mathrm{Dr}^{\mathrm{a}}$ Maria de Lourdes Tavares da Silva e ao Dr. José Nunes de Sousa pelas sugestões e orientação científica.

\section{REFERÊNCIAS BIBLIOGRÁFICAS}

1. Oates DJ, Berlowitz DR, Glickman ME, Silliman RA, Borzecki AM. Blood pressure and survival in the oldest old. J Am Geriatr Soc. 2007;55(3): 383-8.

2. Bejan-Angoulvant $T$, Saadatian-Elahi M, Wright JM, Schron EB, Lindholm $\mathrm{MH}$, Fagard R, et al. Treatment of hypertension in patients 80 years and older: the lower the better? A meta-analysis of randomized controlled trials. J Hypertens. 2010;28(7):1366-72.

3. Lloyd-Jones DM, Evans JC, Levy D. Hypertension in adults across the age spectrum current outcomes and control in the community. JAMA. 2005;294(4):466-72.

4. Beckett NS, Peters R, Fletcher AE, Staessen JA, Lisheng L, Dumitrascu $D$, et al. Treatment of hypertension in patients 80 years of age or older. N Engl J Med. 2008;358(18):1887-98.

5. Law MR, Morris JK, Wald NJ. Use of blood pressure lowering drugs in the prevention of cardiovascular disease: meta-analysis of 147 randomised trials in the context of expectations from prospective epidemiological studies. BMJ. 2009;338:b1665.

6. Beckett N, Peters R, Tuomilehto J, Swift C, Sever P, Potter J, et al. Immediate and late benefits of treating very elderly people with hyper- 
tension: results from active treatment extension to hypertension in the very elderly randomised controlled trial. BMJ. 2012;344:d7541.

7. Aronow WS, Fleg JL, Pepine CJ, Artinian NT, Bakris G, Brown AS, et al. ACCF/AHA 2011 expert consensus document on hypertension in the elderly: a report of the American College of Cardiology Foundation Task Force on Clinical Expert Consensus Documents. Circulation. 2011;123(21):2434-506.

8. Mancia G, Fagard R, Narkiewicz K, Redón J, Zanchetti A, Böhm M, et al. 2013 ESH/ESC Guidelines for the management of arterial hypertension: the Task Force for the management of arterial hypertension of the European Society of Hypertension (ESH) and of the European Society of Cardiology (ESC). J Hypertens. 2013;31(7):1281-357.

\section{CONFLITOS DE INTERESSE}

As autoras declaram não ter conflitos de interesse.

\author{
ENDEREÇO PARA CORRESPONDÊNCIA \\ Teresa Moura Bastos \\ Praceta Ramalho Ortigão, 166, 4420-289 Gondomar \\ E-mail: mourabastosteresa@gmail.com
}

Recebido em 24-05-2015

Aceite para publicação em 02-01-2016

Artigo escrito ao abrigo do novo acordo ortográfico.

\section{ABSTRACT}

\section{HYPERTENSION IN THE VERY ELDERLY:TO TREAT OR NOT TO TREAT?}

Introduction: Hypertension is the most prevalent cardiovascular risk factor. A decrease in blood pressure (BP) in hypertensive patients below the age of 65 reduces the risk of cardiovascular events and death. There are few studies of the reduction of risk in the very elderly (above 80 years of age).

Objectives: To review the evidence for the reduction of risk of major cardiovascular events and mortality in very elderly patients with anti-hypertensive treatment.

Data source: National Guideline Clearinghouse, Guideline Finder, Canadian Medical Association, The Cochrane Database, DARE, Bandolier, MEDLINE/PubMed e Índex de Revistas Médicas Portuguesas.

Methods: A search was conducted on MEDLINE and evidence-based medical sites for articles published between January 2004 and August 2014 in English, Spanish or Portuguese using the keywords 'Hypertension/therapy' AND 'Aged, 80 and over'.

Results: Eight articles met the inclusion criteria. In the guidelines found, antihypertensive treatment reduced the incidence of stroke and cardiovascular morbidity and increased mortality from other causes (Strength of Recommendation C). For patients in good physical and psychological condition, BP values should be around 140-150mmHg (Strength of Recommendation B). Otherwise, treatment should be individualized and monitored clinically (Strength of Recommendation C). One meta-analysis found a reduction in the risk of stroke, cardiovascular events and heart failure (LE 2). Clinical trials show benefit in controlling BP to $150 / 80 \mathrm{mmHg}$ (LE 1). Lower values are associated with greater risk of death and heart failure (LE 2).

Conclusion: The overall benefit of treatment of elevated blood pressure in very elderly is questionable. Although it is associated with a reduction in the risk of cardiovascular events, all-cause mortality, and cause-specific mortality, the results are mixed (Strength of Recommendation B). The reduction of all-cause mortality was achieved in studies with lower reductions of blood pressure and less intensive therapies (Strength of Recommendation B).

Keywords: Hypertension/therapy; Aged, 80 and over 